\title{
IV. Symposium of Young Researchers on Pharmaceutical Technology, Biotechnology and Regulatory Science
}

January 19-21, 2022 - Szeged, Hungary

DOI: $10.14232 /$ syrptbrs.2022.30

\section{The effects of chemical permeation enhancer glycols on the skin}

Nikolett Kis ${ }^{1}$, Maria Gunnarsson², Emma Sparr², Anita Kovács ${ }^{1}$, Szilvia Berkó ${ }^{1}$

\footnotetext{
${ }^{1}$ University of Szeged, Faculty of Pharmacy, Institute of Pharmaceutical Technology and Regulatory Affairs, Szeged, Hungary

${ }^{2}$ Lund University, Chemistry Department, Division of Physical Chemistry, Lund, Sweden
}

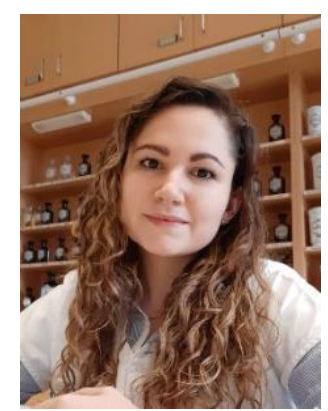

The skin ensures an attractive alternative administration route to conventional drug formulations. However, the stratum corneum (SC), the outermost layer of the skin, forms a barrier against skin permeation. Chemical permeation enhancers are widely used compounds to overcome the barrier function of the skin. They influence the SC structure, which may enhance the dermal drug permeation.

During this research work, we examined three glycols to define their effects on the SC structure and to associate these effects to their permeation enhancer properties. We used NMR spectroscopy to characterize the molecular mobility of SC compounds, and Raman spectroscopy to examine the permeation of a model drug into the different skin layers.

The results from NMR measurements showed that the glycols increased the mobility of SC lipid components, and they also had effects on the keratin filaments. Furthermore, a saturation level for all glycols could be seen, after which the addition of chemicals did not increase the mobility of SC components. The results of Raman spectroscopy showed a significant permeation enhancer effect using the glycols in the formulations, thereby the molecular and the permeation enhancer effect of the examined glycols correlated well during the research.

References

1. Pham D., Björklund S., Engblom J., Topgaard D., Sparr E. J Control Release. 232, 175-187 (2016)

2. Björklund S., Andersson J.M., Pham Q.D., Nowacka A., Topgaard D., Sparr E. Soft Matter 10, 4535-4546 (2014)

\section{Acknowledgements}

This work was supported by the Gedeon Richter's Talentum Foundation (Gyömrői street 1921., Budapest, Hungary, 1103), the construction EFOP 3.6.3-VEKOP-16-2017-00009, and ERASMUS+ mobility program with Lund University. 